\title{
Beiträge zur Kenntniss der persistirenden Pupillarmembran.
}

(Mitgetheilt aus der Augenklinik des Dr. Bolesl. Wicherkiewicz zu Posen.)

Von

Dr. Bogdan Wichorkiewicz.

Hierzu Taf. II. III, Fig. 1-11.

Bekanntlich besteht während des foetalen Lebens eine Membran, welche die ganze vordere Fläche der Linse überzieht, die sogenannte Pupillarmembran.

In welchem Monate diese Membran beim menschlichen Foetus auftritt, ist noch nicht ganz sicher bekannt. Verschiedene Forscher, wie z. B. Wrisberg, Cloquet und Held nehmen den dritten bis vierten Monat an. Zu dieser Zeit jedoch, wo die Membran die Linsenfläche überzieht, bestehen weder Iris noch vordere Augenkammer. Die Entwickelung der Pupillarmembran geht demnach derjenigen der Iris voraus.

Früher glaubte man, dass die Pupillenmembran vom Pupillarrande der Iris ausgehe, mit der Iris in einer Ebene verlaufe und dann die Pupille verdecke. Diese Ansicht vertraten beispielsweise Wachendorf, Haller und $\mathrm{Z}$ inn. RudoIphi wies jedoch nach, dass die Membran vor der Iris liege, welcher Befund auch später durch zahlreiche Untersuchungen von Henle bestätigt wurde. Henle (9) zeigte, dass die Mernbran von der vorderen Irisfläche aus 
an dex Grenze der Sphineterpartie entspringe, der Pupillarrand aber stets frei hinter der Pupillarmembran liege; die Pupillarmembran erhalte vom Pupillarrande keinerlei Verbindungen.

Man kann sich die Sache folgendermassen vorstellen: Die Membran liegt vor der Entwickelung der Iris als vordere Wand der membrana capsulo-pupillaris, mit welcher sie einen gefässhäutigen, das Linsensystem überall eng umschliessenden Sack bildet, der Vorderfläche der Linse an. Beim Hineinwachsen der Iris gegen die Linse hebt sie jene Wand ab, doch bleibt der Pupillartheil derselben nach wie vor auf der linse liegen.

Deber die Zeit, in welcher die Membran schwindet, sind die Ansichten ebenfalls getheilt. Einige Aatoren beobachteten sie noch völlig im siebenten Monate, wie z. B. Cloquet, andere bis zum Ende des intrauterinen Lebens, wie z. B. von Ammon, noch andere sogar in den ersten Monaten post partum.

Der Rückbildungsprocess besteht darin, dass sich die Gefässe dieser foetalen Membran, welche sich in Form von Areaden ausbreiten, gegen den Pupillarrand zurückziehen und dann verengern, wonach das Bindegewebsstratum verödet. Bleiben aber noch einige Reste zurück, so bildet dies eben eine pathologische Erscheinung.

Man sprieht gewöhnlich von persistirender Pupillarmembran, während es sich doch nicht immer um den pupillaren Theil allein handelt, denn es bleibt oft auch mebr von der foetalen Gefasshaut zurück. Die Uncorrectheit dieser Auffassung betont Bock (1) in der April-Nummer der Kl. Mbl. d. J., p. 168, wenn er sagt: „Es blieb erst neueren Untersuchungen vorbehalten, reeht nachdrücklich zu betonen, dass man besser von einer Membrana capsulopupillaris, als ron einer Membrana pupillaris sprechen müsse. Residuen der Membrana pupillaris allein gehen immer fadenförmig ron der Vorderfläche der Iris aus, meist 
aus der Sphinctergegend. Sind aber Theile der der Vorderkapsel der Linse anhaftenden Membrana capsulo-pupillaris nicht involvirt, sondern stehen geblieben, so findet man nicht nur die Adhaerenz der oben erwähnten Fäden an der vorderen Linsenkapsel, sondern auch eine mehr oder weniger reiche Pigmentirung der Linsenkapsel."

Allein auch diese Eintheilung scheint uns nicht stichhaltig zu sein; vielmehr sollte der auf der Linse haftende oder innerhalb der Pupille sichtbare Theil als Membrana pupillaris, der auf der Iris auflagernde als der iridische Theil der foetalen Kapselhaut bezeichnet werden.

Was veranlasst aber nun die Verödung dieser gefässhaltigen Häute? Diese Frage beantworten einige Forscher so, dass sie Contractionen dex Iris annehmen, welche zur Obliteration der Gefässe führen, andere glauben, dass sich neue Gefässsysteme bilden, wie beispielsweise das der processus ciliares, wodurch die Blutzufuhr für die schon bestehenden Gefässsysteme stets geringer wird, und die Gefässe so allmählig veröden müssen.

Es scheint der Mangel an Blutzufuhr das wesentlichste Moment für das Verschwinden der Membran zu sein. Man will in dieser Beziehung verschiedene Injectionsversuche gemacht und auch bald nach der Geburt injicirte Gefässe gefunden haben. Doch sind die Angaben hierüber so spärlich und so unzuverlässig, dass man daraufhin nichts. Sicheres behaupten kann. So hat zwar Tiedemann (23) bei einem ausgetragenen todtgeborenen Kinde die stehen gebliebene Pupillarmembran injicirt, giebt aber über das Resultat dieser Injection keinen Aufschluss.

Die Hauptquelle für die Blutgefässe der Pupillarmembran soll nach Henle (9) im sogenannten Circulus iridis internus liegen, und soll das Häutchen eine grosse Anzahl von Gefässen besitzen. Sie entspringen als radiär verlaufende Aestehen, theilen sich, anastomosiren mit einander vielfach und bilden so ein reiches Capillarnetz. Im 
vorigen Jahre lieferte auch Rumszewicz (17) dafür den Beweis und zeigte, dass nur die venösen Wege fur die Pupillarmembran und für das Gefässsystem des Glaskörpers gemeinsam sind, welche Ansicht bereits von Virchow. (24) Lieberkühn (14) und Konigstein (13) ausgesprochen worden ist. Mit Unrecht leiten daher Einige die Gefasse der Pupillarmembran von der Art. hyaloidea s. capsularis des Foetus ab.

Hinsichtlich der Form, in welcher die Ueberreste der Pupillarmembran auftreten, sind diese meistentheils dickere oder dûnnere Fäden. Sie gehen von den Bogen oder Zacken des Circulus iridis minor aus, überspringen den Pupillarrand und treten zur vorderen Kapsel, wo sie isolirt bleiben oder mehrere zusammen eine Vereinigung bilden. In manchen Fällen besteht nur ein einziger Faden, wie z. B. in dem Falle, welchen Brière (2) zu beobachten Gelegenheit hatte. Es ging dort von den Radiärfasern der Iris ein ganz feiner Faden aus und war vor der Pupille ausgespannt. Die Linsenkapsel berührte er nirgends.

Ein anderesmal krommen wiederum mehrere Fäden vor, bis zu zwolf and mehr. Ihr Verlauf bis zur vorderen Kapsel zeigt ebenfalls Verschiedenheiten; denn einmal verlaufen sie bis an den Pupillarrand isolirt, ein anderesmal haben sie schon vorher Ramificationen und gegenseitige Anastomosen gebildet. Sehr oft befindet sich in der Mitte der Linsenkapsel eine unregelmässige, verschieden gefärbte Platte, welche nicht selten mit der Kapsel verklebt ist.

Ueber solehen Fall berichtet z. B. Schöler (20): Die Membran hing als centrale weisse Platte durch feine Fäden mit der Vorderfläche der Iris zusammen. (Die Anomalie bestand auf beiden Augen.)

Ferner sah Hirschler (10) die Pupillarmembranreste in Form unregelmässig runder Platten auf der Linsenkapsel, welche durch einzelne Fäden mit dem Papillarrande, zusammenhingen. (Die Affection betraf beide Augen.) 
Schapringer (19) sah einen Fall, wo die Pupillarmembran am linken Auge als eine Scheibe erschien; am rechten Auge bestand eine solche Mydriasis, dass die Iris auf einen $1 \frac{1}{2} \mathrm{~mm}$ breiten Streifen reducirt war.

In einem Falle, den Talko (22) beschreibt, war mit der vorderen Kapsel eine braune Platte verwachsen, zu der die Fuden von der Vorderfläche der Iris gingen. Die Pupille war frei beweglich. (Affection an beiden Augen.)

Ueber einen ahnlichen Fall referirte Cohn (3). Auch dort gingen zur Mitte der Linsenkapsel, auf der eine $1,5 \mathrm{~mm}$ breite Platte lag, zahlreiche feine Fäden von der Irisfläche, und die Beweglichkeit der Pupille war in keiner Weise behindert. (Die Anomalie betraf beide Augen.)

v. Hasner (8) publicirte einen.Fall, wo sich in der Mitte der Linsenkapsel eine kleine membranöse Platte befand, die durch einen sich dann mehrmals theilenden Faden mit der vorderen Fläche der Iris in Verbindung stand. Die Hornhaut hatte einen Durchmesser von nur 9,5 mm. Das Auge war stark myopisch, und waren im Innern desselben atrophische Veränderungen nachzuweisen. - Die membranöse Platte wurde dann operativ entfernt, wonach sich die Sehschärfe ganz bedeutend besserte. Die Linse war ohne jegliche Trübung und die Iris frei beweglich. (Beide Augen waren afficirt).

In einem zweiten ebenfalls von v. Hasner (7) operativ angegriffenen Falle lag eine mohnkorngrosse, gelbliche Platte auf der Linsenkapsel. Von dieser Platte ging zum mittleren, äusseren Irisquadranten ein Faden, der in sechs feinere Fäden zerfiel. Diese sechs Fäden inserirten sich an der äusseren Grenze der Circulus iridis internus.

Einen ganz besonderen Fall bekam Klein (12) zur Beobachtung. Die Membran entsprang ziemlich peripherisch in der Mitte der Irisbreite, war dabei geschlossen und berührte nirgends die Kapsel. (Anomalie beiderseits.)

Mayerhausen (15), Graefe (6), Szokalski (21) 
und van Duyse (4) berichten über ganz ähnliche Fälle. Verschieden von solchen ist der von Hirschberg (11) beobachtete. Bei gewöhnlicher Beleuchtung konnte man nur ein paar weisse Punkte im Pupillargebiet erkennen. Bei focaler Beleuchtung dagegen sah man, dass die mittlere Zone des Pupillarkreises von einem sehr zierlichen Netze, dessen dunklere Fäserchen enge Masehen bildeten, eingenommon war. Das Centrum blieb frei, und in der peripheren Zone waren zwischen Irisrand und dem Netze einige etwas stärkere Fäden ausgespannt. (Affection beiderseits.)

Die Pupillarmembranen sind meist sehr zart und enthalten gewohnlich gar kein Pigment. Im Gegensatz hierzu findet man mitunter die Pupillarmembranen bei Erwachsenen entweder pigmentirt oder sie sind ziemlich dick, auch zusammengesetzt aus verhältnissmässig dicken Fäden. Es bängt dies ab von der abnormen Entwickelung der Membran selbst während des fötalen Lebens. Rumszewicz spricht die Ansicht aus, es sei möglich, dass in den Fällen, in welchen die Papillarmembranreste eine Pigmentirung zeigen, dies von der abnormen Differenzirung der Keimblätter der mittleren Platte der Linsenkapsel abhängen könne, zumaI dann, wenn sich an diesen Stellen Pigmentzellen entwickeln. Dort wiederum, wo die Pupillarmembranreste farblos sind, könnten dies die sich gleich anfangs stärker entwickelnden Adventitiae der Gefassse versehulden.

Bekanntlich entstehen nämlich aus den Kopfplatten in dem vorderen Theil des Bulbus die Cornea (ohne ihr Epithel), die Iris mit der Pupillenmembran und die Linsenkapsel. Von diesen Theilen enthält nun bei normaler Entwickelung nur die Iris ein Pigment. Bei abnormer Entwickelung findet sich das Pigment-Epithel nicht nur im Umfange der Pupillenmembran, sondern sogar auf der Linsenkapsel. Jedenfalls wurde bis jetzt die hintere Linsenkapsel stets ungetrübt gefunden, anch sind im Glaskörper 
nie Gefässreste angetroffen worden. Es ist daher dieser constante negative Befund ein Beweis, dass der vordere und hintere Theil der gefässhaltigen fötalen Linsenkapsel unabhängig sind oder aber anderen Bedingungen für den Rückbildungsprocess unterworfen sind. Der negative Befund würde nach den bisherigen Untersuchungen allerdings noch nicht dafür sprechen, dass es unter keinen Umständen Reste der hinteren fôtalen Gefässkapsel der Linse geben könne; vielmehr glaubt mein Bruder, dass mancher hintere Kapselstaar als etwas der persistirenden Pupillarmembran Analoges angesehen werden dürfte. Sicheren Aufschluss hierüber kann allerdings erst eine gründliche histologische Untersuchung eines einschlägigen Falles geben.

Es sollen die Pupillarmembranreste öfter an einem als an beiden Augen angetroffen worden sein. $\mathrm{Ob}$ auch die Heredität hierbei in Frage kommt, ist nicht sicher bekannt. Jedenfalls ist es auffallend, dass in gewissen Fallen mehrere Kinder derselben Eltern mit dieser Anomalie behaftet sind. Wir werden uns auch davon z. B. in dreien später zu schildernden Fällen überzeugen können.

Was die Statistik der persistirenden Membranen anlangt, so wurden seit dem Jahre 1881, bis zu weleher Zeit nur 30 Fälle beschrieben waren, über 100 neue publicirt. Es kann daher auffallend sein, dass gerade die letzten Jahre so ansehnliche Zahlen aufweisen. Aber sehr richtig bemerkt van Duyse (4) gleich im Beginne seiner Abhandlung, dass diese Fülle der publicirten Fälle von der subjectiven Anschauung abhängen möge, die ein jeder Beobachter von der Sache hatte. Viele rechneten nämlich zn diesen Missbildungen nicht nur die ausgesprochenen membranösen Reste, sondern auch isolirte Irisfäden, ja selbst Pigment-Auflagerungen auf der vorderen Kapsel, welche nicht einer entwickelten Iritis zugeschrieben werden konnten.

Man hat wohl zuweilen entzündliche Auflagerungen 
innerhalb der Pupille für embryonale Missbildungen gehalten, doch ofters dürfte das Umgekehrte stattgefunden haben, und mag man letztere gerade für Entzündungsproducte angesehen und ihnen nicht die nöthige Beachtung geschenkt haben. Dies würde wenigstens den Umstand erklären, weshalb man früher diesen Missbildungen so selten begegnet sein sollte.

Die differenzial-diagnostischen Merkmale sind schon von A.d. Weber (5) eingehend hervorgehoben worden, so dass ich dieselben hier übergehen kann.

Die Functionsstörungen, die durch diese Reste verursacht werden, haben keinen bestimmten Charakter und richten sich nach der Form der centralen Endigungen der Fäden, nach der Grösse, Form und Ausdehnung der Membran u. s. w. In vielen Fällen beeinträchtigt der meist unvollständige Verschluss der Pupille das Sehvermögen und die Accommodation nur wenig. Franke hat in 32,5 pCt. Fällen von persistirenden Membranen Astigmatismus gefunden und spricht die Ansicht aus, derselbe könne möglicherweise in Folge der zugleich mit der Cornea gemeinsam sich entwickelnden Pupillarmembran entstehen.

Man unterlässt es daher für gewöhnlich, durch einen operativen Eingriff die Membran zu entfernen. Dort aber, wo die Platte einen grossen Theil des Pupillargebiets verdeokt und das Sehvermögen in bedeutendem Maasse alterirt, schreitet man zur operativen Behandlung, wie dies beispielsweise Graefe, Cohn, v. Hasner, van Duyse, Rumszewicz und mein Bruder gethan haben.

In anatomischer Hinsicht hat $\mathrm{P}$ onfick zuerst die Membranreste untersucht und zwar in einem von H. Cohn (3) beobachteten Falle, in dem ein Stück der Regenbogenhaut und der Pupillarmembran durch eine Iridectomie herausgeschnitten worden war.

Die Fäden der Membran bildeten ein dichtes Fasergewebe, welches ein wenig wellig war und von reichen 
von den Irisgefässen herstammenden Capillaren durchzogen. Nur an wenigen Stellen konnte man auf beiden Seiten Epithelbelag nachweisen. Die $2 \mathrm{~mm}$ langen Fäden entsprangen in einer Entfernung von $1 / 3 \mathrm{~mm}$ vom Pupillarrande. Auf beiden Flächen wurde hier und dort etwas Pigment angetroffen.

Ausserdem verdanken wir van Duyse (4) eine recht genaue Untersuchung, welche der genannte Forscher der Beschreibung seines zweiten Falles beifügt.

Membranreste, die von Leichen entfernt worden waren, haben Wedl und Bock vor zwei Jahren mikroskopisch untersucht. Die pigmentirten Stränge erwiesen sich als collabirte Gefässe mit Andeatung eines Lumens und einer dicken, Pigmentzellen einschliessenden bindegewebigen Scheide; in ihrer Insertionsstelle an der vorderen Linsenkapsel bafteten vollständig entwickelte, mit einem hellen Kern und mehreren Fortsätzen versehene abgeflachte Pigmentzellen.

Beim ersten Fall, den van Duyse in seiner Abhandlung beschreibt, bedeckte die Membran die Pupille des rechten Anges nnd die Faden gehen von dieser Membran aus nach dem peripherischen Theile der Iris weit über den Circulus iridis minor.

Der genannte Beobachter betont nun ausdrücklich, dass dies eine Ausnahme bei derartigen Anomalien sei. Nach den von meinem Bruder in dieser Richtung angestellten Untersuchungen, die ich in seiner Klinik mehrmals zu verfolgen Gelegenheit hatte, sehienen aber diese Ausnahmen gar nicht so selten vorzukommen, was übrigens auch der Ansicht Rumszewicz's und Michel's (16) entspricht. Wenn man nämlich bei focaler Beleuchtung von der Seite, namentlich mit einer Loupe, die Irisflache genau beobachtet, dann kamn man sich in vielon Fällen überzeugen, dass die persistirenden Fäden, ehe sie sich mit dem Irisgewebe vereinigt haben, nach der Peripherie zu in Form eines Dreiecks 
breiter werden. Ein solches Dreieck besteht aus lauter kleinen Fädchen, die mit einander vielfache Anastomosen eingehen, und sieht wie ein kleinmaschiges Netz aus. Sehr oft hat man nun Gelegenheit, derartige kleine Netze im ganzen Umfange der geschlängelten Linien zu bemerken in Iriden, die sonst keine persistirende Faden aufweisen. Aber noch deutlicher kann man es dann verfolgen, wonn dies restirende Pupillarnetz in stärkerem Grade vorhanden ist und stellenweise wirkliche Nembranreste darstellt. Durch Vergleichung der Niveaudifferenzen zwischen den mit diesen Membranen zusammenliegenden Faden, die bis an die Peripherie reichen, und dem zwischen den Maschen des Pupillarnetzes sichtbaren Irisgewebe tritt dieses Verhältniss noeh erkennbarer hervor.

In dieser Hinsicht leistet die Westien-Zehender'sche Loupe Vorzügliches. In mehreren unserer Fälle, die wir weiter unten besehreiben werden und die wir mit der oben genannten Loupe untersucht haben, werden wir sehen, dass die Fäden sogar bis zur Corneoscleralgrenze reichen. Ehe wir jedoch zur Schilderung unserer Falle schreiten, will ich an dieser Stelle das Wesentlichste aus den drei interessanten Fällen, die Rumszewicz (18) im vorigen Jahre publicirt hat, und die in Vielem den unsrigen ähneln, hervorheben. Wir halten uns dazu um so mehr berechtigt, als jene Arbeit, da sie in poInischer Sprache veröffentlicht ist, wohl wenig den deutschen Aerzten zugänglich sein dürfte.

Fall 1. E. N., 21 Jahre alter Mann. Rechtes Auge: Auf der Linsenkapsel befindet sich eine braune, fast runde Platte ron $2 \mathrm{~mm}$ Durchmesser, welehe ziemlich stark nach vorne tritt. Oberhalb der Mitte der Platte und neben deren oberem und äusserem Rande sieht man zwei gabelförmig sich theilende Fadenzüge. Zwischen dem Pupillarrande und der Platte liegen die Fäden lose in der vorderen Augenkammer und vereinigen sich mit dem Irisgewebe $1 \mathrm{~mm}$ vom Pupillarrande entfernt. Linse, Glaskörper, Gefässe und Augenhintergrund sind normal; 
wohl aber sind Zeichen von Astigmatismus irregularis vorhanden; $\mathrm{S}=5 / 200$.

Linkes Auge: Hier war die Platte auf der Mitte der Kapsel unregelmässig geformt, doch ziemlich so gross wie rechts. Die äussere Hälfte der Pupille war von Fäden durchkreuzt. Der mittlere dünne Theil der Fäden haftete an dem ausseren Rande der Platte auf der Linsenkapsel. Linse, Glaskörper und Augenhintergrund waren normal. $S=15 / 200$.

Fall 2. B. K., 21 Jahr alter Mann. Rechtes Auge: Oberhalb der Mitte des Pupillargebiets war eine Platte von der Form eines unregelmässigen Vierecks. Von dieser Platte gingen 5 Gewebszüge nach verschiedenen Richtungen aus, vor allen aber 2 nach oben, 2 nach aussen und 1 nach unten. Der letztere wurde in der Gegend des Pupillarrandes breiter und theilte sich dann in weitere Fäden. Diese äzsserst zarten, farblosen Fäden sind mit der Kapsel nicht verwachsen, und vereinigen sich, was hier gerade hervorzuheben ist, erst in der Ciliarzone mit der Iris. Auch die Platte war mit der Kapsel nicht verwachsen und sehien gleichsam an den Fäden in der vorderen Augenkammer aufgehängt zu sein. Linse, Glaskörper und Angenhintergrund waren völlig normal. $\mathrm{S}={ }^{20} / 40$. Astigmatismus hyperopieus.

Linkes Auge: Auf der Linsenkapsel erschien eine unregelmässig geformte, hellbraune, dentlich nach vorne gebogene Platte. An verschiedenen Stellen konnte man auf derselben dunkle Fadenzüge erkennen. Die auch hier farblosen Fäden inserirten sich ebenfalls erst ganz in der Nähe des Ciliartheils der Regenbogenhaut. Der Augenhintergrund konnte nicht gut untersucht werden, weil die Platte einen grossen Theil des Pupillargebietes verdeckte. $\mathrm{S}={ }^{20} / 200$.

Fall 3. N. S., 20 Jahre alter Mann. Das rechte Auge ist vollig normal. Linkes Auge: Auf der Vorderfläche der Linsenkapsel präsentirte sich eine ovale, dunkelbraune Platte, die in der Mlitte ca. $1 \mathrm{~mm}$ nach vorne gewölbt erschien; in horizontaler Richtung war sie $2 \mathrm{~mm}$ lang und ca. $1,5 \mathrm{~mm}$ hoch. Durch die mehr nach oben zu gerichtete Platte war bei starker Myosis die obere Hälfte des Pupillargebiets eingenommen. Vom unteren Plattenrande liefen nach unten hin sechs dunkelbraune Fäden strahlenförmig ans, welche sich später vielfach theilten und was wir hier wiederum betonen wollen, fast an den Ciliartheil der Iris herangingen. Da die Sehschärfe kaum $4 / 2$ oo betrug, 
so machte Rumszewicz zur Verbesserung der'selben nach unten hin oine künstliche Pupille. Mit der Liobreich'schen Pincotte riss er die mit der Platte nur lose vereinigten Fäden ab und erhielt sie im Zusammenhange mit der fast unverletzten Nembran und dem ausgeschnittenen Irisstück. Hinter der Platte war die Kapsel sowie die Linse völlig transparent. Die künstliche Pupille war $1,5 \mathrm{~mm}$ breit. Nach der Operation betrug $\mathrm{S}={ }^{20} / 100$.

Rumszewicz behauptet, dass wie beimFötus die Pupillarmembran bald näher bald weiter rom Pupillarrande an die lris fixirt ist, was vollkommen individuell und nicht vom Alter des Fötus abhängig ist, anch bei Erwachsenen die Fäden der Pupillarmembran einmal eben noch den Pupillarrand erreichen, ein anderes Mal wiederum bis an den Ciliartheil der Iris herantreten könnten. Das Letztere beobachteten z. B. Graefe und Szokalski.

Wird man aber nunmehr die etwa zur Beobachtung seitens der Aerzte gelangenden Fälle mit der binocularen Loupe, wie das in den von uns zu analysirenden Fallen gesehehen ist, genau untersuchen, so dürten sich, wie bereits oben bemerkt, viel mebr solcher persistirenden Membranen herausstellen, bei denen die Fäden bis an den äussersten Ciliartheil der Iris zu verfolgen sind.

Nach diesen einleitenden Bemerkungen wollen wir an die Beschreibung der von meinem Bruder beobachteten Fälle der genannten Anomalie herantreten. Wir werden genauer auf drei davon eingehen, vorerst aber noch kurz einige solche besprechen, die weder den Namen einer Pupillarmembran verdienen, noch auch irgendwie das Sehvermögen alteriren, đie jedoch einen Beweis dafür abgeben, dass Reste der fotalen Gefässhant am mensehlichen Auge häufiger vorkommen und in der mannigfachsten Form auftreten können. Ganz minimale Reste kommen gewöhnlich nur zufällig zur Beobachtung. 
Fall 1.

Dieser Fall ist dem von Briere beschriebenen ziemlich analog und verdankt seine Entdeckung einem Fremdkörper.

C. K., Sehlosserlehrling, 15 Jahre alt. Bei der Entfernung eines Fremdkörpers bemerkte mein Bruder einen ganz feinen Faden im linken Auge. Dieser Faden hatte das Colorit der Iris, trat ganz in der Nähe des Pupillarrandes aus dem Irisgewebe hervor und ging später in einem nach unten convexen Bogen temporalwärts und weiter nach oben, wo er sich wieder im Irisgewebe am oberen Pupillarrande inserirte (Fig. 1). Nach der Einträufelung von Atropin dilatirte sich die Pupille gleichmässig, und der Faden wurde ganz straff, wie eine Violinsaite auseinandergedehnt (Fig. 2).

\section{Fall 2.}

16jähriger, junger Mann mit Strabismus convergens, Mikrophthalmos und Nebulae corneae. Bei der Untersuchung: constatirte mein Bruder am linken Auge einen kleinen Pupillarmembranrest am äusseren Pupillarrande, welcher in ein äusserst zartes Häutchen überging und den unteren Theil des Papillargebiets verdeckte.

\section{Fall 3.}

M. P., ein 23 jährigs Mädchen, mit rechtsseitiger Bindehautentzündung. Rechts Phthisis bulbi. Die Cornea hatte einen Durchmesser einer Erbse. Am linken Auge zogen rom unteren Pupillarrande zur Mitte der Pupille ganz zarte Fädchen, die von demselben Colorit wie das der Iris waren.

\section{Fall 4.}

Dieser, sowie die beiden folgenden Fälle sind auch insoforn äusserst interessant, als sich diese congenitale Anomalie bei drei Geschwistern vorfand und ferner, dass bei allen dreien Ectopie der Linse und Excentricität der Pupille vorhanden war. Bemerken will ich hier gleich, dass die Eltern in keinem verwandtschaftlichen Verhältnisse zu einander standen und sechs lebende Kinder (4 Töchter und 2 Söhne) besassen.

L. K., ein 7 jähriges Mädchen, wurde vor fünf Jahren der Augenheilanstalt zu Posen wegen Kurzsichtigkeit zugeführt. Der Befund war folgender: Am rechten Ange schlotterte die Tris, die Pupille lag excentrisch nach nnten und zwar so, dass die Iris bei mittlerer Pupillenweite oben $6 \mathrm{~mm}$, unten nur 
$2 \mathrm{~mm}$ broit war. Am linken Auge dagegen lag die Pupille excentrisch nach aussen und oben, und war die Iris nach innen and unten $7 \mathrm{~mm}$, nach aussen $1 \mathrm{~mm}$ breit. Die Hornhautbreite betrug $11 \mathrm{~mm}$

Untersuchung nach Atropinwirkung: Rechtes Auge: die Pupille hat sich zwar bedeutend, ungefizhr auf $6 \mathrm{~mm}$ dilatirt, doch sah der Pupillarrand selur unregelmässig aus, weil ron allen Seiten, vom Rande her ganz kleine Fädehen zur Linse zogen, ihren Ursprung vom Irisgewebe nehmend. Sie waren von gleichem Colorit, wie das der Iris und zweifelsohne Reste einer Pupillarmembran. Das stark myopische Auge liess oplthalmoskopisch nach anssen hin einen atrophischen Bogen der Chorioidea mit einem pigmentirten Rande erkennen.

Linkes Auge: auch hier besteht eine, wenn auch etwas geringere, Mydriasis. Die Pupille ist nach oben und aussen ein wenig excentrisch geblieben. Nau erkennt ebenfalls Fädchen, die jedoch dicker sind und sowohl mit einander, wie auch mit den gegenüberliegenden vom Irisrande ziehenden Fäden in Verbindung treten. Theilweise berühren sie noch kaum die Iris, theilweise sind sie mit derselben verwachsen. Nichts destoweniger erseheint durch dieses Fadennetz der Augenhintergrund roth. Man erkennt den äusseren Linsenrand; die Linse reflectirt stark. Die zwisehen dem Linsen- und Trisrande gelegene freie Stelle beträgt ca. $2 \mathrm{~mm}$. Auch dieses Auge ist myopisch.

Das Mädchen wurde in der Klinik am linken Ange operirt. Mit dem Lanzenmesser wurde ein breiter Schnitt nach oben gemacht; die Iridectomie war regulär, gross und mit einem Scheerenschlage ausgeführt. Hierbei trat eine Glaskörperhernie ein, worauf die Linse in geschlossener Kapsel ohne Glaskörperverlust schnell extrahirt wurde.

Die Linse war dick und klein, jedoch völlig transparent. Bemerken will ich, dass die Operation sehr gestört wurde dureh die während der Narkose sich äusserst unruhig verhaltende kleine Patientin. Monoculus.

Am folgenden Tage wurde der Verband abgenommen; die Wundränder waren mit einander verklebt, die Pupille war rein. Frischer Verband. - Da in den zwei folgenden Tagen die Heilung günstig verlief, so wurde der Verband abgenommen, und Patientin ambulatorisch behandelt. Nach Verlauf von einigen Tagen wurden die Augen nochmals untersucht: Die Pupille des operirten Auges war rein und gross; das Auge 
zeigta keinerlei Reizerscheinungen. Mit Hilfe des Keratoskops erkannte man in der oberen Hornhauthälfte Astigmatismus.

Das Ophthalmoskop wies links Hyperopie ca. 7 D., rechts Myopie ca. 8 D. nach. Rechts zählte Patientin Finger in $5^{\prime}$ ohne Glas, in $20^{\prime}$ mit -4 , links dagegen Finger in $4^{t}$ Entfernung ohne Glas, in $15^{\prime}$ mit +7 .

Nach 8 Tagen bekam sie Gläser (rechts ein Planglas, links +7 D) und wurde entlassen.

\section{Fall 5.}

Bei der jüngeren, 4 Jahre alten Schwester W. K., welche die Eltern mitgebracht hatten, wich die Pupille rechterseits nach aussen und etwas nach unten $\mathrm{ab}$, linkerseits dagegen nach aussen und oben. Nach der Instillation von Atropin zeigte sich folgendes Bild:

Rechtes Auge: Die Pupille hatte sich fast gleichmässig dilatirt; in ihrem Centrum sah man den Irisrand, der nach oben und innen gewichen war. Ueberdies waren hier ebenso wie bei der älteren Schwester Fädchen, jedoch in geringerer Zahl vorhanden, welche an Zartheit denen eines Spinnengewebes glichen und über die Pupille hinwegzogen. Die Refraction war an den linsenfreien Stellen Hyperopie, an den übrigen starke Myopie. Das Ophthalmoskop liess in der Umgebung der Papille eine Atrophie der Chorioidea erkennen. Linkes Auge: gleichmässige Mydriasis, am Irisrande kleine Fädchen, gleich denen am rechten Ange; anch hier war der innere Rand der Iris mehr nach vorne gerückt, und die linse nach oben und innen gewichen, so dass der äussere Linsemrand die Pupille halbirte. Mit dem Ophthalmoskop erkennt man ein Staphyloma posticum von der Grösse einer halben Papille mit pigmentirtem Rande. Das Auge war myopisch resp. hyperopisch.

\section{Fall 6.}

Im Oktober desselben Jahres brachten dieselben Eltern ein drittes, 2 Jahre altes, sehr mittelmässig entwickeltes Kind M. K. in die Klinik. Die Lider öfnete es nur ungern; die Iriden waren in lebhafter Bewegung. Rechterseits lag die Pupille nach oben hin excentrisch, die linse nach aussen; nasalwärts war die Iris grösser und nach vorne gerichtet. Linkerseits war die Pupille ebenso excentrisch nach aussen und etwas nach unten gelegen; die innere obere Hälfte der Iris trat nach

v. Graefe's Archiv tum Oplithalmologie, XXXIV. 4. 
vorne. Die Pupillen waren unregelmässig rund, die radiären Faserzüge waren an beiden Augen sehr gut erkennbar.

Die Augen wurden atropinisirt, wonach rechts die Pupille $5 \mathrm{~mm}$ lang und $4 \mathrm{~mm}$ breit erschien. Die Iris dieses Auges hatte oben aussen eine Breito von ea. $2 \mathrm{~mm}$, unten von ca. $4 \mathrm{~mm}$. Links war die Pupille mehr oder weniger rund, lag excentrisch und hatte einen Durchmesser von $5 \mathrm{~mm}$. An beiden Augen war in der Mitte der Papille der Rand der Linse (rechts der obere, links der untere äussere) sichtbar: letztere war nach hinten zu luxirt und stand mit dem Ciliarkörper in keinerlei Verbindung. Die Linse bedingte eine bedentende Myopie; in dem linsenfreien Theile aber bestand Eyperopie. Die Umgebung der Papille war ein wenig atropisch, und man sah hier und da Pigmentablagerungen.

Vom Pupillarrande gingen theils zum gegenüberliegenden, theils zum benachbarten Rande äusserst zarte Fäden, die man erst mit Hilfe einer Loupe deutlich zu erkennen vermochte. Doch sehien kein Faden die Linsenkapsel zu erreichen. - Das Kind wurde nach Hause geschickt, und sollte in einigen Jahren wieder vorgestellt werden, was aber bis jetzt noch nicht geschehen ist.

Die nunmehr folgenden drei letzten Fülle aus der Clientel meines Bruders haben wir Dank der ausgezeichneten Zehender'schen Loupe einer minutiösen Untersuchung unterziehen können and sind daher im Stande, eine genauere Beschreibung derselben zu geben, der wir mehrere selbst gefertigte Zeichnungen beifügen.

\section{Fall 7.}

Gelegentlich einer systematischen Schulenuntersuchung auf Granulose bemerkte mein Bruder bei einem siebenjährigen Knaben, A. K., eine persistirende Pupillarmembran an beiden Augen. Die Untersuchung ergab Folgendes:

Rechtes Auge: Die Pupille ist ein klein wenig excentrisch nach oben gelegen; die Tris hat eine gelbbrame Farbe. Anf der Vorderfläche derselben sieht man eine gelbbraune Membran (Fig. 3), die, an mehreren Stellen durchiöchert, fast bis an den Ciliarrand der Regenbogenhaut reicht und zum Theil die letztere durchschimmern lässt. Diese Membran, welche in den peri- 
pheren Theilen der Iris fest anliegt, hebt sich in der Gegend des Circulus iridis major von der Vorderfäche der Iris $a b$ und den Charakter einer Membran verlierend, geht sie in einzelne Füden über, welche je mehr nach dem Pupillarrande sich um so mehr nach vorne wenden, miteinander anastomisiren und frei in der vorderen Augenkammer flottiren. Einzelne dieser Fäden and zwar drei stärkere und ein feinerer, wenden sich in einem stark nach vorne convexen Bogen zur Vorderfläche der Kapsel, an welcher man eine feine die Mitte und den oberen Theil einer mässig verengten Pupille einnehmende Kapselauflagerung vorfindet, welche mit diesen Fäden verbunden ist. Bei Erweiterung der Pupille bemerkt man unregelmässig eoncentrische Faltenbildnngen in dem peripheren Theile der Pupillarmembran, durch die man aber andererseits auch die Faltenbildungen der Iris hindurchschimmern sieht. Eine genaue Abgrenzung der Pupillarmembran im peripheren Theile ist gar nicht gegeben, weil die Membran bis in den Winkel der Vorderkammer reicht,

Linkes Auge: Auch hier ist die Pupille ein wenig nach oben und nasalwärts excentrisch gelegen. Man findet hier ganz analoge Verhältnisse wie am rechten Auge, nur sind die Maschen, durch die man das freie Pupillargebiet sieht, noch etwas kleiner als am rechten Auge. Die Pupillarmembran ist in der Gegend des Circulus iridis major mit dem Irisgewebe scheinbar eng verfilzt und geht von hier aus in eine ganz feine, homogene, durchschimmernde Haut über, während sie sich pupillarwärts vom Cir. iridis major an abhebt und in stärkere und feinere Fäden, die mit einander vielfach anastomosiren, übergeht. Unter diesen Fäden markiren sich vor allen diejenigen, die von oben und von unten kommen, da sie ihrerseits aus etwas stärkeren Fäden, die mit einander ein wirkliches Maschennetz bilden, zusammengesetzt sind und sich, wie am rechten Auge, in einem nach vorne convexen Bogen zur vorderen Kapsel wenden. Beide netzförmigen Schlingen (also die obere und untere) befestigen sich an je eine von einander durch ein horizontales linienformiges freies Pupillargebiet getrennte graue Kapselauflagernngen, welche die mässig verengte Pupille fast gänzlich verdecken. Der Pupillarrand ist ebenso wie am rechten Auge vollständig frei und das Pupillenspiel unbehindert.

$\mathrm{S}$ rechts $=6 / 9$. Em. (Schw. 0,4). S links $6 / 36-24$. M. $1.5 \mathrm{D}$. (Sch. 0,8). Nach Instillation von Atropin war $\mathrm{S}$ links $=6 / 24 \mathrm{Hp}$. 2 D. Die Pupille hatte sich gleichmässig dilatirt. Die Unter- 
suchung mit dem Keratoskop zeigte nichts Abnormes, anch der ophthalmoskopische Befund war normal.

Am 8. Mai 1887 wurde die Dislaceratio membranae pupillaris oculi sinistri ansgeführt. Cocain. Antiseptik mit Sublimat.

Durch einen Längenschnitt nach oben, $1 \mathrm{~mm}$ unterhalb des Hornhautrandes, führte der Operateur das Irishäckchen ein, löste damit den unteren Theil der Membran, riss den oberen Theil $a b$ und führte ihn aus dem Ange heraus, worauf derselbe am Häekehen durchsehnitten, nnd die Iris reponirt wurde. Eserin. Binoculus.

Am 10. Mai. Das Auge zeigt nicht die geringsten Reizerscheinungen; die Iris ist frej beweglich, rund, und in ihrem oberen Theile fehlt die Pupillarmembran vollstandig. Atropin.

14. Mai. Das Ange ist völlig reizlos, and sind nur noch wenige Conjunctivalgefäss $\theta$ injicirt. Die Pupille erscheint frei, nur unten besteht ein minimaler Membranrest. $S$ links $=6 / 24$ 且 $1,5 \mathrm{D}$.

21. Mai. Mit der Loupe sieht man auf der Iris in ihrem oberen Theile den kleinen Rest, von dem die Membran abgelöst worden ist. Unten berührt der Membranrest die Kapsel nicht. $\mathrm{S}$ links $=6 / 18$. Ast. hyp. $1,5 \mathrm{D}$. Axe vert. Sch. 0,5. Der Augenhintergrund ist völlig normal.

27. Juni. Das Pupillargebiet ist gänzlich froi (Fig. 4); an der Kapsel ist weder bei focaler Beleuchtung, noch beim Ophthalmoskopiren irgend welche Trübung nachzuweisen. Das obere Schlingennetz ist ganz verschwunden; man sieht nur in der Gegend des Circulus iridis major einzelne abgeschnittene, frei in die vordere Kammer hineinragende Fadenstücke, während das untere Fadennetz gleichfalls von der Kapsel abgetrennt ist, etwas verkürzt frei flottirt und bei mässig erweiterter Pupille den Pupillarrand überragt. $\mathrm{S}$ links $=6 / 18$. Sch. 0,4 .

Das Sehvermoggen hat sich demnach bedeutend gebessert, und der Knabe wird aus der Behandlung entlassen. Diese Zunahme der Sehkraft hat sich aber in der Folge noch stärker erwiesen. Im Februar d. J. wurde nämlich der Knabe zur Klinik citirt; auch untersuchten wir noch einmal seine Augen; das Keratoskop wies eine der Schnittführung entsprechende anomale Hornhautkrümmung nach, und es war rechts, $\mathrm{S}={ }^{20} / 20$, H. 2 D, J. 1, links, $\mathrm{S}={ }^{20} / 50-40$, Ast. hyp. $0,75 \mathrm{D}$, J. 1.

Das herausgeschnittene äusserst feine Stück der Pupillar- 
membran wurde zunächst in Glycerin auf einem Objectglase auseinandergefaltet, geebnet und unter schwacher Vergrösserung untersucht. Man konnte so nur ein an beiden Seiten scharf abgeschnittenes Netz von leicht pigmentirtem Gewebe und gleichmässiger Strulktur erkennen (Fig. 10). Nach Monaten wurde das winzige Präparat aus dem Glycerin herausgenommen, mit Hämatoxylin und Eosin gefärbt und sowobl nnter schwacher als auch starker Vergrösserung untersucht. Man sieht deutlich als Grundsubstanz reichliche Bindegewebszellen (Fig. 11), von denen einige pigmentirt sind, getragen von einem Fasergewebe. Durch die ganze Membran ziehen feine Capillaren, erkennbar an der Intima und dem Endothel (Eosin). Ausserdem sind hier und da einzelne Blutlörperchen, auch Pflasterepithel, zu erkennen.

\section{Fall 8.}

J. M., ein Knabe von 6 Jahren. Wemn schon der vorhergehende Fall einen Beweis abgeben konnte, dass die Pupillenmembran nicht allein in einzelnen Fäden post partum, sondern unter Umständen als eine vollständige zusammenbängende Membran sich noch lange Zeit erhalten kann, so giebt der nächstfolgende Fall in weit höhorem Grade einen Beweis dafür ab.

Rechtes Auge: Vor allen Dingen ist es gerade dieses Auge, welches in dieser Hinsicht ein seltenes Exemplar darbietet. Hier finden wir (Fig. 5) fast die ganze Iris und die ganze Pupille durch eine graugelbe Membran verdeckt, die allerdings an verschiedenen Stellen, namentlich aber innerhalb des Pupillargebiets und über der Papillarzone der Iris, durchlöehert erseheint. Vom Circulus iridis major an ist die Membran von der Irisfläche abgehoben und mit einer ziemlich starken Convexität nach vorne gerichtet. Man erkennt zwar an den stärkeren Oeffnungen der Pupillarmembran, dass diese Haut mit der Iris in der Gegend des Cireulus iridis major rerfilzt ist, doch ist bei genauerer Betrachtung zu sehen, dass die Pupillarmembran an der genannten Stelle ihre Existenz nicht aufgiebt, sondern dass sie sich anch weiter nach der Peripherie hin ausdehnt, hier gleichmässig die Ciliarzone der Iris bedeckt und nur an einzelnen Stellen, besonders jedoch temporalwärts, in feine Fäden endigt. Es ist dies nicht nur bei richtiger Einstellung der Westien-Zehender'schen Loupe für versehiedene Tiefen zu beobachten, sondern auch daran, dass die Pupillarzone der Iris, deren freie Bewegung durch die durchlöcherten 
Stellen in der Membran zu verfolgen sind, dunkelbraun er* scheinen, während die Ciliarzone dureh die gleichmässige Pupillarmembran verdeckt im ungleichmässigen Blau hindurehschimmert.

Soweit man die Papille durch die feinen Membranöffnungen überblicken konnte, war an der Kapsel keine Trübung zu erkennen. Entsprechend der Pupillenverdeckung war auch die Sehkraft dieses Auges ganz bedeniend vermindert, denn der Knabe konnte rechts nur auf $20^{\circ}$ Finger zählen.

Linkes Auge: Die Pupillarmembran weicht insofern von der des rechten Auges ab, als hier zunächst die rom Circulus iridis major sich abhebenden Fäden viel stärker und zahlreicher sind und dadurch Raum für die dem Pupillartheile der Iris entsprechenden Oeffnungen in der Membran geschaffen ist. Das Gebiet der durchlöeherten Membran geht allnählig in einen zusanmenbängenden Membrantheil über, an dom man allerdings Linien erkennt, die sich stärker markiren (Fig. 6). Diese zusammenhängende Membran bedeckt den temporalen Theil der Pupille and befestigt sich an die Mitte der Kapsel. Der an die Kapsel befestigte Theil der Membran entspricht dem Umfange einer ziemlich stark verengten Pupille (etwa $1^{1 / 2}-2 \mathrm{~mm}$ im Durchmesser). Das Pupillarspiel ist ebenso wie bei den vorhergehenden Fällen, gänzlich frei, und es ist interessant, zu beobachten, wie sich bei stark verengter Pupille die Pupillarmembran in Falten um die Kapseltrübung legt und die Pupille gänzlich bedeckt.

Im Allgemeinen sind also an der Pupillarmembran des linken Auges drei Theile zu unterscheiden, und zwar: der periphere oder ciliare, der über dem ciliaren Theile der Regenbogenhaut gleichmässig ausgespannt ist, der intermediäre, fadenförmige oder maschennetzartige, der am Circulus iridis major beginnt und einige Millimeter breit pupillarwärts hinzieht und der membranartige Pupillartheil, der die Fortsetzung des intermediären bildet und dessen nasaler Theil an die Mitte der vorderen Kapsel angeklebt ist.

Dass es sich hicr nicht um eine Kapseltrübung im eigentlichen Sinne des Wortes handelt, also um eine entzündliche Ausschwitzung und Verdickung der Linsenkapsel, sondern dass wir es hier mit einer Verklebung der Pupillarmembran mit der normalen Linsenkapsel zu thnn haben, können wir aus den beiden operirten Fallen ersehen. Es gelang nämlich, wie wir 
es schion gesehen und aus der Beschreibung der folgenden Operation gleich sehen werden, die Membran von der Kapsel leicht zu trennen, ohne dass nachträglich, selbst bei Loupenuntersuchung, irgend welche Trübung der Linse zu bemerken gewesen wäre, ein Moment, welches hier hervorgehoben werden muss.

Die Sehkraft des linken Auges betrug ${ }^{20} / 50-40$. Um die Sehkraft des rechten Auges zu heben, schlug mein Bruder den Eltern eine Operation vor, die am 23. Juni 1886 in folgender Weise von ihm ausgeführt wurde:

Nachdem alle antiseptischen Vorbereitungen getroffen waren, wurde der Schnitt mit dem Lanzenmesser nach oben hin geführt, worauf der Operateur mit dem Irishäkchen die Membran zu entfernen suchte. Diese riss jedoch, trotzdem sie wiederholt gefasst wurde, durch. Da das Pupillargebiet frei geworden war, so wurde die etwas vorgefallene Iris reponirt und ein Verband angelegt.

24. Juni. Das Auge ist ohne jegliche Reizung, die Wundränder verklebt, liegen gut an, die Iris ist in ihrem oberen Theile etwas mehr der Hornhaut genähert, das Centrum der Pupille ist frei. Atropin-Instillation und frischer Verband.

25. Juni. Der Heilungsyerlauf ist äusserst günstig, die Pupille hat sich sehr gut dilatirt, im Centrum und oben ist sie frei. Atropin und Monoculus.

26. Juni. Die Pupille hat sich heute noch stärker ausgedehnt. Im Uebrigen Status idem. Atropin.

Einige Wochen später wurde die Sehkraft dieses Auges untersucht; es zeigte sich hierbei, dass $\mathrm{S}$ rechts $={ }^{20} / 40 \mathrm{H} 1 \mathrm{D}$. war. Die ein Jahr später vorgenommene Untersuchung dieses Anges ergab folgendes Resultat:

Ueber dem verticalen Meridian der Cornea sieht man an der Corneoscleralgrenze eine $3 \mathrm{~mm}$ breite lineare Narbe, die sich etwa $1^{1 / 2} \mathrm{~mm}$ cornealwarts fortsetzt und mit der Ciliarzone der Iris resp. der Pupillarmembran an einer Stelle verwachsen ist. Die Pupille (Fig. 7) selbst ist rund und frei beweglich, bei starker Verengerung gänzlich frei, bei mässiger Verengerung an der Peripherie verdeckt durch die nach der Operation zurückgebliebenen retrahirten peripheren Theile der frei in die vordere Kammer hineinragenden Pupillarmembran. Sonst hat sich die Pupillarmombran in ihrem Aussehen nicht geändert.

Nur wäre noch hervorzuheben, dass man mit der binocu- 
laren Loupe genau übersehen kann, dass nicht die Regenbogenhaut, sondern die die Ciliarzone derselben überziehende feine, spinnwebenartige Membran mit der Operationsnarbe verwachsen und rom Irisgewebe abgehoben ist.

\section{Fall 9.}

F. M., Nähterin, 46 Jahre alt, kam im April d. J. mit ihrem an einer leichten Augenentzündung leidenden kleinen Kinde zur Klinik. Mein Bruder, dem die Frau solbst zu gleicher Zeit über zeitweise eintretende Müdigkeit im rechten Auge klagte, entdeckte hier eine persistirende Pupillarmembran, die sich in einer äusserst interessanten Weise präsentirte.

Ich habe von diesem Falle mit Hilfe der Zehender'schen Loupe zwei Zeichnungen (Fig. 8 und Fig. 9) entworfen, die ich der Beschreibung beifüge:

Die Pupillarmembran ist ebenso wie die Regenbogenhaut von dunkelbrauner Farbe (Fig. 8) und scheint in den peripheren Theilen eng mit dem Trisgewebe verfilzt zu sein. In der Gegend des Circulus iridis major hebt sie sich allmählig von der Irisfläche ab und, indem sie mit einer ziemlich starken Convexität nach vorne gerichtet ist, endigt sie in der Gegend des Circulus iridis minor. Diese Endigungen haben im oberen inneren und äusseren Irisquadranten einen zungenförmigen Charakter, im äusseren unteren Quadranten dagegen bemerken wir ungefähr vier bis fünf von der Membran ausgehende Fortsătze, die ihrerseits in zwei im Bogen nach links und rechts auslaufenden kurzen Fädchen endigen. Einige von diesen Fädchen sind mit einander verbunden. In der Mitte des inneren unteren Quadranten läuft die Membran in zwei warzenähnliche Theile aus, die jedoch zusammen verwachsen sind und auf der Pupillargrenze mit einer starken Convexität sich nach vorne wenden und späterhin in einen zungenformigen Fortsatz auslaufen. Dieser Fortsatz reicht fast bis zur Mitte des Pupillargebiets und flottirt bei genauer Betrachtung mit der oben genannten Loupe in der vorderen Angenkammer. Von der Spitze dieses Fortsatzes geht im Verlauf des verticalen Augenmeridians ein ganz feiner Faden aus, der, nachdem er eine Zweitheilung eingegangen, sich in die Membran einpflanzt. Temporalwärts von diesem Faden besteht im Pupillargebiet ein etwa stecknadelkopfgrosser Fleck. Nasalwärts von der Spitze der oberen Wurzel des oben beschriebenen warzenförmigen Fortsatzes be- 
merkt man einen äusserst feinen, kurzen, fadenförmigen Fortsatz. Rechts $\mathrm{S}={ }^{20} / 40-30$ Ast. h. 1,5 D. 45 t. J. 3. Links $\mathrm{S}={ }^{20} / 20$ Hp. 0,5. J. 1 c. $+1,5$.

Die Untersuchung mit dem Keratoskop ergab bei der schiefen nasalen Axe eine Verlängerung um 1-11/2 mm. Ophthalmoskopisch bestand beiderseits Hyperopie. Die Papillen waren von einem feinen Pigmentsaume umgeben.

Nach der Instillation von Eserin ins rechte Auge verkleinerte sich, wie Fig. 9 zeigt, das Pupillargebiet dieses Auges ziemlich stark. Die Pupillargrenze war jedoch keineswegs kreisrund, sondern oval, weil durch die Verwachsungen der warzenähnlichen Fortsätze der Membran mit dem Irisgewebe die Sphincterthätigkeit an dieser Stelle beeinträchtigt war.

\section{Literatur - Verzeichniss.}

1. Bock. Klin. Honatsbl. für Augenheillkunde, April 1888: Pigmentklümpchen in der Vorderkammer frei beweglich.

2. Brière. Observations eliniques 1874; Ann. d'oculist. t. 72, p. 110.

3. Cohn, H. Zur Anatomie der persistirenden Pupillarmembran. Centralbl. f. Augenheilkunde, April 1881.

4. van Duyse. Contribution à l'étude des membranes pupillaires persistantes. 1886.

5. Graefe, Alfr. Arch. f. Ophth. VIII.

6. - - ibidem XI. 1, p. 208: Ueber membr. pup. pers. and Polycorie. (1865.)

7. v. Hasner. Operative Entfernung der persistirenden Pupillarmembran durch Korelyse, Prag. med. Wochenschrift 1883, No. 47 u. 48.

8. - - Wiener Centralbl. f. pr. Augenheilk. 1884, p. 32.

9. Henle, J. De membrana pup. aliisque membranis pellucentibus. Diss. Bonn 1832.

10. Hirschler. Ein Fall von Membr. pup. pers. Szemészet. Beilage zum Orrase Hetilap 1874, No. 1.

11. Hirschberg. Erster Bericht über seine Augenklinik. Berliner klin. Wochenschrift, p. 528 etc. 1870.

12. Klein. Ueber einen Fall von persistirender Pupillarmembran. Wien. med. Presse, No. 31 u. ff. 1878.

13. Koenigstein. Ueber die Pupillarmembran. Archiv für Ophthalmol, XVII. 3, p. 60. 
14. Lieberkühn. Arch. f. Anatomie u. Physiologie 1879.

15. Mayerhausen. Klin. Monatsbl. f. Angenheilk, Jan. 1886, p. 17.

16. Michel. Arch. f. Ophthalm. XXVII, 2, p. 205.

17. Rumszewicz. Pamiętniki Towarzystwa przyrodników $w$ Kijowie 1887.

18. - - . Trzy nowe przypadki przetrwania błony źrenicznéj. Przeglad lekarski, No. 36, 1887.

19. Schapringer, A. Mydriasis, persistent pupillary membrane, Med. and surg. Reporter, July 11, 1874.

20. Schoeler, H. Jahresbericht über seine Augenklinik. Berlin 1874.

21. Talko, J. Klinische Monatsblätter für Augenheilk. 1871, p. 230.

22. - - Ein Fall von Membr. pup. pers, utriusque oculi. Ibid. 1882, S. 346.

23. Tiedemann und Treviranus. Zeitschr. f. Physiologie, Bd. II. Heft 1, 1827.

24. Virchow. Würzburger Sitzungsberichte, 24. Mai 1879.

\section{Erklärung der Abbildungen.}

Fig. 1. Reste der Pupillarmembran, Fall 1, bei natürlicher Weite der Pupille.

Fig. 2. Dasselbe nach Atropinwirkung.

Fig. 3. Reste der Pupillarmembran, Fall 7, rechtes Auge.

Fig. 4. Fall 7, linkes Auge, nach operativer Entfernung der persistirenden Pupillarmembran.

Fig. 5. Persistirende Pupillarmembran, Fall 8, rechtes Auge.

Fig. 6. Dasselbe, Fall 8, linkes Auge.

Fig. 7. Fall 8, rechtes Auge, nach operativer Beseitigung der Pupillarmembran.

Fig. 8. Persistirende Pupillarmembran, Fall 9, bei natürlicher Weite der Pupille.

Fig. 9. Dasselbe, nach Eserinwirkung.

Fig. 10. Extrabirte Pupillarmembran von Fall 7, Glyceriupräparat.

Fig. 11. Ein Stïck derselben Pupillarmembran, nach Haematoxylinfärbung. 


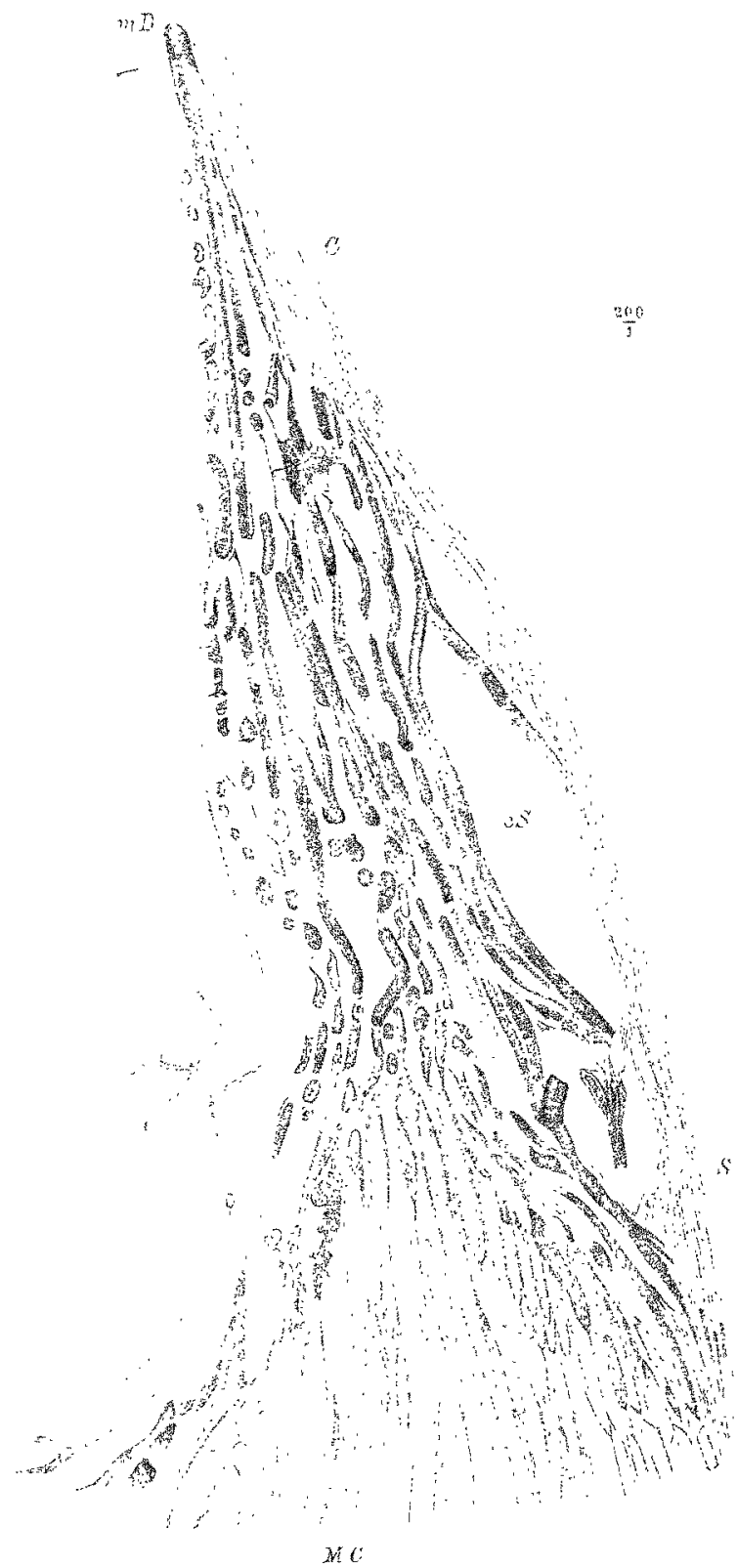



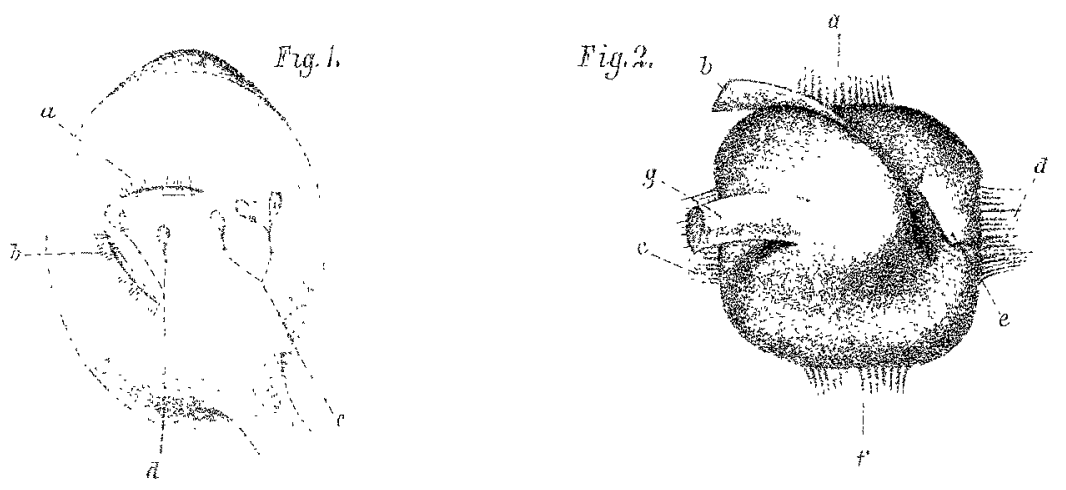

Lign.

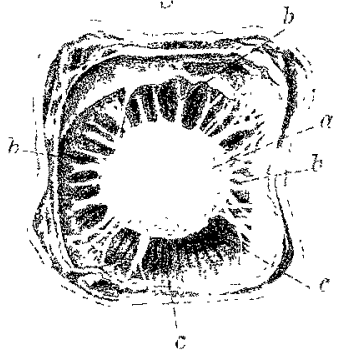

Fig. 4

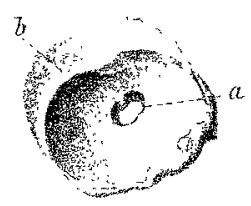

Fig, io.

sin

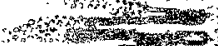

Zs

$\because 3$

$L_{-w}$

ifs

nos

Froil

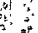

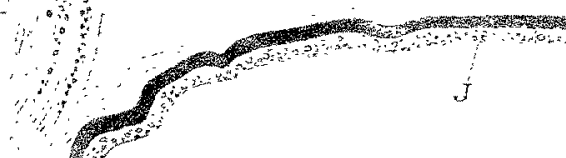

(19)

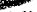

